\title{
Stochastic Scheduling With Inertia-Dependent Fast Frequency Response Requirements
}

Fei Teng, Student Member, IEEE, Vincenzo Trovato, Student Member, IEEE, and Goran Strbac, Member, IEEE

\begin{abstract}
High penetration of wind generation will increase the requirement for fast frequency response services as currently wind plants do not provide inertial response. Although the importance of inertia reduction has been widely recognized, its impact on the system scheduling has not been fully investigated. In this context, this paper proposes a novel mixed integer linear programming (MILP) formulation for stochastic unit commitment that optimizes system operation by simultaneously scheduling energy production, standing/spinning reserves and inertia-dependent fast frequency response in light of uncertainties associated with wind production and generation outages. Post-fault dynamic frequency requirements, 1) rate of change of frequency, 2) frequency nadir and 3) quasi-steady-state frequency are formulated as MILP constraints by using the simplified model of system dynamics. Moreover the proposed methodology enables the impact of wind uncertainty on system inertia to be considered. Case studies are carried out on the 2030 Great Britain system to demonstrate the importance of incorporating inertia-dependent fast frequency response in the stochastic scheduling and to indicate the potential for the proposed model to inform reviews of grid codes associated with fast frequency response and future development of inertia-related market.
\end{abstract}

Index Terms-Frequency control, power system dynamics, stochastic programming, unit commitment, wind integration.

\section{NOMENCLATURE}

\section{A. Constants}

$\Delta \tau(n)$

$\eta_{s}^{c} / \eta_{s}^{d}$

$\mu(j)$

$\pi(n)$

$\sigma$

Time interval corresponding to node $\mathrm{n}(\mathrm{h})$. Charge/discharge efficiency for storage unit $s$. Diurnal adjustment constant corresponding to the $j$ th time step of the day. Probability of reaching node $n$ Standard deviation of random Gaussian increments in autoregressive time series. $\sigma_{z}(i)$ Standard deviation of forecast error in normalized wind level, $i$ time steps ahead.

$\varphi_{1}, \varphi_{2}$ $A(n)$ $\mathcal{G}$ Autoregressive parameters.

Set of nodes that are ancestors of node $n$. Set of thermal generators.

Manuscript received November 21, 2014; revised March 21, 2015; accepted May 11, 2015. This work was supported by the UK's Engineering and Physical Sciences Research Council under Grant EP/L014351/1 and EP/L014386/1. Paper no. TPWRS-01591-2014.

Color versions of one or more of the figures in this paper are available online at http://ieeexplore.ieee.org.

Digital Object Identifier 10.1109/TPWRS.2015.2434837
$\mathcal{S}$

$\mathcal{N}$

$a(n)$

$c^{\mathrm{LS}}$

$c_{g}^{m}$

$c_{g}^{\mathrm{nl}}$

$c_{g}^{\mathrm{st}}$

$P_{g}^{\max }$

$P^{\text {ming }}$

$P_{g}^{\text {msg }}$

$T_{g}^{\text {mo }}$

$T_{g}^{\mathrm{mu}}$

$T_{g}^{\mathrm{st}}$

$P_{s}^{c \max }$

$P_{s}^{d \max }$

$E_{s}^{\max }$

$E_{s}^{\min }$

$R_{g, s}^{\max }$

$f_{g}^{F}$

$H_{g}$

$H_{L}^{\max }$

$D$

$T_{d}$

$R o C o F_{\max }$

$f_{0}$

$\Delta f_{\max }$

$\Delta f_{\max }^{\mathrm{ss}}$

$\Delta f_{\mathrm{DB}}$

$q(n)$

$W(\cdot)$

$X(k)$
Set of storage units.

Set of nodes on the scenarios tree.

Parent node of node $n$.

Value of lost load ( $\mathcal{L} \mathrm{MWh}$ ).

Marginal cost of thermal unit $g\left(\mathcal{L}^{\prime} \mathrm{MWh}\right)$.

No-load cost of thermal unit $g(\mathcal{L} / \mathrm{h})$.

Startup cost of thermal unit $g(\mathcal{L})$.

Maximum generation of thermal unit $g(\mathrm{MW})$.

Minimum stable generation of thermal unit $g$ (MW).

Minimum off time of thermal unit $g(\mathrm{~h})$.

Minimum up time of thermal unit $g(\mathrm{~h})$.

Startup time of thermal unit $g(\mathrm{~h})$.

Maximum charge rate of storage unit $s$ (MW)

Maximum discharge rate of storage unit $s$

(MW)

Maximum charge level of storage unit $s$

(MWh).

Minimum charge level of storage unit $s$

(MWh).

Maximum frequency response capability of

thermal unit $g$ or storage unit $s$ (MW).

Proportion of the spinning headroom, which can contribute to frequency response provision. Inertia constant of thermal unit $g(\mathrm{~s})$.

Inertia constant of tripped generator (s).

Load damping rate $(1 / \mathrm{Hz})$

Delivery time of frequency response (s)

Maximum rate of change of frequency $(\mathrm{Hz} / \mathrm{s})$.

Nominal frequency level (Hz)

Maximum frequency deviation requirement in Nadir (Hz).

Maximum frequency deviation requirement at quasi steady state $(\mathrm{Hz})$.

Frequency deadband of governor $(\mathrm{Hz})$

Forecast error quantile of branch leading to node $n$.

Sigmoid-shaped function which transforms the wind level to an aggregated wind output. $k$ th element in an autoregressive time series which represents normalized wind level. 


\section{B. Semi-Constants (Fixed With Respect to Linear Program} But Variable Between Timesteps)

$P^{D}(n) \quad$ Total demand at node $n(\mathrm{MW})$.

$P^{\mathrm{WN}}(n)$ Total available wind generation at node $n(\mathrm{MW})$.

\section{Decision Variables}

$P^{\mathrm{LS}}(n) \quad$ Load shed at node $n(\mathrm{MW})$.

$P^{\mathrm{WC}}(n)$ Wind curtailment at node $n(\mathrm{MW})$.

$N_{g}^{\text {sd }}(n) \quad$ Number of thermal unit $g$ that are shut down at node $n$.

$N_{g}^{\text {st }}(n) \quad$ Number of thermal unit $g$ that are started up at node $n$.

$P_{g}(n) \quad$ Power output of thermal unit $g$ at node $n(\mathrm{MW})$.

$N_{s}^{\text {Gen }}(n)$ Operation status (0/1 for Pumping/Generating) of storage unit $s$ at node $n$.

$P_{s}^{c}(n) \quad$ Charge rate of storage unit $s$ at node $n(\mathrm{MW})$

$P_{s}^{d}(n) \quad$ Discharge rate of storage unit $s$ at node $n$ (MW)

$R_{g, s}(n) \quad$ Primary frequency response provision from thermal unit $g$ or storage unit $s$ at node $n$ (MW).

\section{Linear Expression}

$N_{g}^{\text {up }}(n) \quad$ Number of thermal unit $g$ that are online at node $n$.

$N_{g}^{\text {off }}(n) \quad$ Number of thermal unit $g$ that are offline at node $n$.

$N_{g}^{\mathrm{sg}}(n) \quad$ Number of thermal unit $g$ that start generating at node $n$.

$C_{g}(n) \quad$ Operating cost of thermal unit $g$ at node $n(\mathcal{L})$

$E_{s}(n) \quad$ State of change of storage unit I at node n (MWh)

$H(n) \quad$ System inertia after generation loss at node $n$ (MWs/Hz).

$R(n) \quad$ Total Frequency response provision at node $n$ (MW).

\section{INTRODUCTION}

$\mathbf{I}$ NTEGRATION of large share of wind generation increases the system requirements for various ancillary services, which will be mostly delivered by part-loaded generators in combination with fast standing plants. This will not only decrease the system operation efficiency and consequently increase operation cost, but it may eventually compromise the system ability to integrate wind generation. In particular, the lack of system inertia exacerbates the need for fast frequency response services [including both inertia response and primary frequency response (PFR)] in order to maintain the frequency evolution within security boundaries and avoid, in the worst case, emergency demand disconnections. In fact, the lack of inertia already causes wind curtailment in Ireland [1], [2], which limits the maximum instantaneous system nonsynchronous penetration ratio at $50 \%$.

At present, ancillary services are scheduled following deterministic rules by imposing pre-defined requirements in the generation scheduling. As the uncertainty introduced by wind generation is more significant than that by demand scheduling process performed under such deterministic rules may be inefficient [3] Stochastic optimization with mixed integer linear programming (MILP) has been applied to schedule operating reserves to deal with various sources of uncertainty [4], [5]. Scenario reduction techniques are investigated in [5] and [6] to alleviate the computational burden of stochastic programming. Moreover, a full stochastic unit commitment (SUC) model is proposed in [7], which also optimally schedules PFR. However, the impact of the level of system inertia on the scheduling of PFR has not been explicitly modeled in SUC.

In fact, there has been significant interest in directly incorporating post-fault frequency requirements in economic dispatch (ED) and unit commitment (UC) models. The authors in [8] proposed a MILP formulation for UC with frequency regulation constraints. The provision of PFR from each generator is modeled as a linear function of frequency deviation, covering only quasi-steady-state frequency deviation in most cases. Doerthy et al. introduced frequency control in the generation dispatch model [9]. Nonlinear frequency constraints are derived by performing a number of dynamic simulations to ensure the rate of change of frequency (RoCoF) and the frequency deviation within the security thresholds. Linear constraints on frequency are developed in [10] to ensure the frequency response adequacy. However, the load damping effect is not considered and system inertia is assumed to be known and not variable. A more recent work [11] incorporated analytical nonlinear frequency constraints into a deterministic UC problem. Finally, a frequency-constrained stochastic ED model was developed in [12] to incorporate wind uncertainty and frequency constraints. The results demonstrate the dramatic impact of system inertia on the system operation. However, the UC decision is fixed and the constraints to limit the post-fault frequency are nonlinear.

In this context, this paper proposes an MILP formulation for SUC that directly takes into account of post-fault frequency evolution and guarantees system security against largest plant outage. We identify three key contributions from this paper:

1) The introduction of a novel MILP formulation for system frequency constraints that ensure the dynamic evolution of post-fault frequency to be within limits associated with the RoCoF, nadir frequency and quasi-steady-state frequency (in accordance with the GB security standards [13]).

2) Direct quantification of the impact of wind uncertainty on the system inertia through integrating the constraints associated with the dynamic frequency evolution into the SUC; hence the generation is optimally scheduled across the time scale from seconds to hours.

3) The application of the developed model to the GB 2030 system in order to understand the impact of:

- the degradation in system inertia,

- delivery time of frequency response,

- changing maximum RoCoF level, and

- load-damping rates

on the system operation cost and the ability of the system to integrate wind generation. The proposed scheduling framework could inform 1) reviews of grid codes associated with fast frequency response and 2) the development of inertia-related market, particularly in systems with significant contribution of wind generation.

The rest of this paper is organized as follows: Section II introduces the stochastic scheduling model. Section III describes 


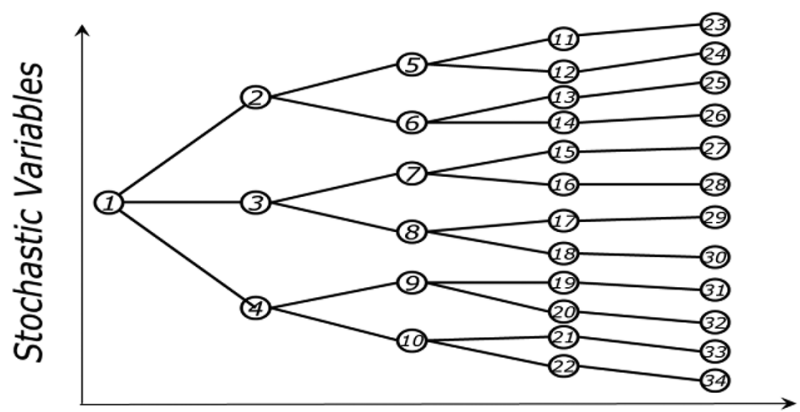

Time ahead

Fig. 1. Schematic of a typical scenario tree in SUC.

the inertia-dependent fast frequency response requirements. The case studies are presented and explained in Section IV, while Section V concludes the paper.

\section{Stochastic Scheduling Model}

A multi-stage stochastic scheduling model is formulated in order to optimally schedule energy production and delivery a number of ancillary services in light of uncertainties associated with wind generation and plant outrages. The UC and ED are solved over a multi-stage scenario tree (Fig. 1). The simulations are carried out with rolling planning approach [5], performing a complete SUC calculation with a 24-h horizon in half-hourly timestep, and discarding all decisions beyond the root node ones. In the next time step, realizations of some uncertain variables become available, which may be different from any existing scenario. An updated scenario tree covering 24-h time horizon is then built; UC and ED decisions are adjusted and inter-temporal constraints are maintained.

\section{A. Scenario Tree}

Quantile-based scenario selection method proposed in [7] and [14] is applied in this paper. This method constructs and weights scenario trees based on user-defined quantiles of the distribution of the net demand. Compared with commonly used Monte Carlo methods, this method captures critical information about the uncertainties by considering only a relatively small number of scenarios. The scenario generation process includes two steps: 1) creation of net demand distribution and 2) calculation of nodal value of net demand and associated probability.

The model in [15] is applied to simulate the wind output and the associated uncertainty. The normalized wind level $X(k)$ is assumed to follow a Gaussian AR(2) process (1) with halfhourly timestep, which is then transformed into a non-Gaussian power output $P^{w}(k)$ with a range from zero to the installed capacity of wind fleet. The parameters are tuned to represent GB system as in [16]:

$$
\begin{gathered}
X(k)=\varphi_{1} X(k-1)+\varphi_{2} X(k-2)+\sigma \epsilon(k), \\
\epsilon(k) \sim N(0,1) \text { i.i.d. } \\
P^{w}(k)=W\left(X(k)+\mu\left(k \bmod N^{d}\right)\right.
\end{gathered}
$$

where $N^{d}$ is the number of timesteps in one day, $W(\cdot)$ is a sigmoid-shaped transformation function, and $\mu(j)$ is used

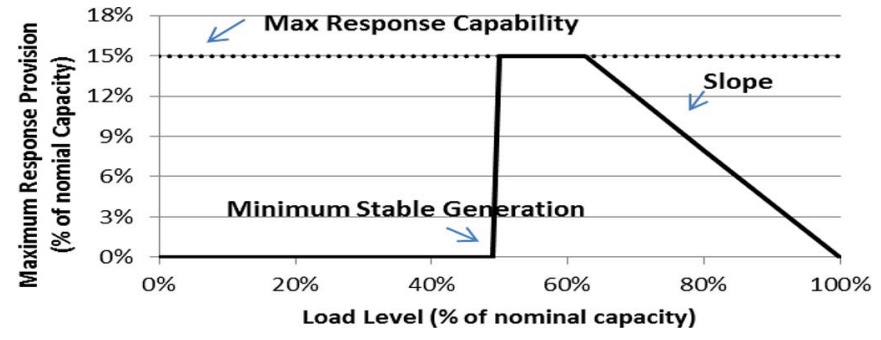

Fig. 2. Example of response characteristic of conventional thermal plants.

to represent a diurnal variation. Generation outages are assumed to follow Markov process [17]. The failure of unit $g$ may occur during each timestep $\Delta t$ with probability $\lambda_{g} \Delta t$ if the unit is online. Unit $g$ that is on outage is repaired with probability $\mu_{g} \Delta t$. The probability distribution of outages is derived by using capacity outage probability table (COPT) [17]. The circulation problem of COPT construction is solved by iterations as proposed in [7]. The cumulative distribution function (CDF) $C(x ; n)$ of the net demand is the total system demand minus the convolution of the probability distribution function (PDF) of realized wind production with the negative cumulative nodal COPT.

Each node $n$ is associated with a user-defined quantile $q(n)$. The $q$ th quantile of the distribution of net demand can calculated as $x: C(x ; n)=q$ by using a numerical root-finding algorithm. The corresponding nodal probabilities $\pi(n)$ in the scenario tree are approximated by using Trapezium rule [14].

\section{B. Stochastic Unit Commitment Formulation}

The scheduling model is a MILP model, which is based on the work presented in [14]. The full mathematical formulation is provided in the Appendix. The objective of the stochastic scheduling is to minimize the expected operation costs, including generation costs and load shedding costs (A1). The optimization subjects to the system-level constraints, including load balance constraint (A2) and fast frequency response constraints (A3)-(A7) (details are discussed in the next section); local constraints for thermal units, such as minimum and maximum generation (A10), commitment time (A11), minimum up and down times (A13)-(A14), ramping rates (A16)-(A17), fast frequency response provision (A18)-(A19), as well as for storage units (A20)-(A25).

Generating units will need to be part-loaded in order to provide sufficient headroom needed to supply PFR. Moreover, due to the generator physical limits, such as governor speed, the maximum amount that each unit could contribute to PFR will be limited, regardless of the loading level [9]. Therefore, as shown in Fig. 2, the amount of PFR that each generator or storage can deliver is limited by its maximum response capability (A16) and the slope $f_{g}^{F}$ that links the frequency response provision with the spinning headroom (A17). Moreover, pump-storage unit is assumed not to be able to shift between pumping and generation mode to provide PFR (A22).

This paper primarily focuses on the scheduling of fast frequency response and transmission network constraints are not considered. 


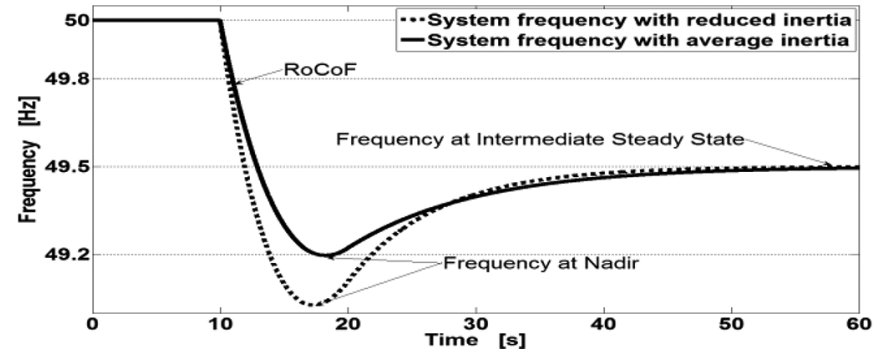

Fig. 3. System frequency evolution after a contingency. The evolution with average inertia (solid) respects the GB security standards, while with reduced system inertia (dotted) these standards may be violated.

\section{Modeling of Inertia Dependent Fast FREQUENCY RESPONSE REQUIREMENTS}

The aim of fast frequency control is to contain the initial dynamic evolution of frequency (e.g., following a generator outage) within defined security thresholds. In GB, this is specified by the Security and Quality of Supply Standard (GB-SQSS) [13]. Three criteria are used to set the security standards for the initial transient evolution of frequency (Fig. 3) in case of the maximum infeed loss:

1) rate of change of frequency (RoCoF);

2) frequency level at Nadir;

3) frequency level at intermediate quasi-steady-state.

The RoCoF achieves the highest absolute value just after the disturbance occurs; initially the frequency drop is only limited by the inertial response of conventional generators; currently the standard prescribes that the RoCoF should not exceed $0.125 \mathrm{~Hz} / \mathrm{s}$ [18]. Furthermore, the PFR has to limit the frequency above the minimum value set to $49.2 \mathrm{~Hz}$. An extended provision of PFR enables meeting the intermediate quasi-steady-state condition; in the case of GB the frequency should stabilize above $49.5 \mathrm{~Hz}$ within $60 \mathrm{~s}$.

The growing concern is the reduced system inertia may compromise the frequency performance as shown in Fig. 3. In particular, the RoCoF will increase, potentially causing disconnections of distributed generators by actuating RoCoF-sensitive protection schemes, which would further exacerbate the problem. In fact, RoCoF relay protection was found to be a main factor limiting the level of penetration of nonsynchronous generation in Ireland [19]. Moreover if the frequency drops rapidly, conventional generators may not be fast enough to provide the scheduled PFR [10]; the resulting frequency nadir may activate the low frequency demand disconnection (LFDD) [13].

\section{A. Dynamic Model of Frequency Evolution}

The time evolution of system frequency deviation can be described by a first order ODE [20]:

$$
2 H \frac{\partial \Delta f(t)}{\partial t}+D * P^{D} * \Delta f(t)=\sum_{g, s \in \mathcal{G}, \mathcal{S}} \Delta P_{g, s}(t)-\Delta P_{L}
$$

where $\mathrm{H}[\mathrm{MWs} / \mathrm{Hz}]$ is the system inertia after generation loss (taking into account the reduction of inertia following the generation outage), $\mathrm{D}[1 / \mathrm{Hz}]$ represents the load damping rate, $\mathrm{P}^{\mathrm{D}}$
[MW] is the load level and $\Delta P_{g, s}$ [MW] describes the additional power provided by the generator $g$ or storage $s$ following the generation loss $\Delta P_{L}$ [MW].

In [10] and [12], a conservative approach is adopted and load damping rate is set at zero, which enables derivation of analytical frequency response constraints. However, ignoring the load damping effect would lead to over-scheduling of the frequency response [21]. In Section IV-D, we demonstrate the level of load damping has a significant impact on the system operation. According to the present GB practice, PFR specifies the power increase to be delivered within $10 \mathrm{~s}\left(T_{d}\right)$ following the contingency [13], while in Ireland the delivery time is $5 \mathrm{~s}$ [9]. The impact of different delivery time requirements is analyzed in Section IV-B. Furthermore, the governor responses are assumed to be linearly increasing with time [10], [12] and thus characterized by a fixed slope until scheduled response is delivered. This model also includes a frequency dead-band $\Delta f_{\mathrm{DB}}$ for the governor [13] that prevents unnecessary response to relatively small frequency deviations. Therefore, the delivery of PFR can be modeled as

$$
\Delta P_{g, s}(t)= \begin{cases}0 & \text { if } t<t_{\mathrm{DB}} \\ \frac{R_{g, s}}{T_{d}} *\left(t-t_{\mathrm{DB}}\right) & \text { if } T_{d}+t_{\mathrm{DB}} \geq t \geq t_{\mathrm{DB}} \\ R_{g, s} & t \geq T_{d}+t_{\mathrm{DB}}\end{cases}
$$

where $t_{\mathrm{DB}}$ represents the time when frequency deviation reaches the dead-band $\Delta f_{\mathrm{DB}}$.

In this paper we propose a formulation to explicitly include the requirements on frequency dynamic evolution within SUC and hence optimally schedule the provision of fast frequency response. For the scheduling of fast frequency response, for simplicity reasons this paper only considers the loss of the largest unit $\left(\Delta \mathrm{P}_{\mathrm{L}}^{\max }\right)$. The differential equation (3) is mapped into SUC model through considering three characteristic periods in the form of constraints associated with the RoCoF, the frequency level at nadir and the frequency level at quasi-steady-state. The proposed constraints below correspond to a single node in the scenario tree; hence the notation for the node $n$ is suspended.

\section{B. Rate of Change of Frequency (RoCoF)}

In the short interval of inertia response, the governors response is negligible (i.e., $\Delta P_{g} \cong 0$ ) as the frequency deviation is small $(\Delta f \cong 0)$. Hence, the maximum value of the rate of change of frequency is proportional to the power shortage and inversely proportional to the system inertia; this suggests that the minimum level of system inertia $H$, required to satisfy the maximum RoCoF requirement is obtained as

$$
\begin{aligned}
H & =\frac{\sum_{g \in \mathcal{G}} H_{g} * P_{g}^{\max } * N_{g}^{\mathrm{up}}-\Delta P_{L}^{\max } * H_{L}^{\max }}{f_{0}} \\
& \geq\left|\frac{\Delta P_{L}^{\max }}{2 R o C o F_{\max }}\right| .
\end{aligned}
$$

\section{Frequency Level at Nadir}

The frequency nadir is defined as the minimum value achieved by system frequency during the transient period. The nadir depends on system inertia and governors' response. The 
system is assumed to be at nominal frequency in the pre-contingency state [10], and the delivery of frequency response is described by (4). By integrating (3), the evolution of frequency deviation is obtained as (6) at the bottom of the page, where $D^{\prime}=D * P^{D}, \Delta P_{L}{ }^{\prime}=\Delta P_{L}^{\max }-D^{\prime} * \Delta f_{\mathrm{DB}}$, and $t^{\prime}=t-t_{\mathrm{DB}}$.

The time $t^{*}$ when the frequency reaches its nadir can be calculated by setting $(\partial|\Delta \mathrm{f}(\mathrm{t})|) /(\partial \mathrm{t})=0$ :

$$
t^{*}=t_{\mathrm{DB}}-\frac{2 H}{D^{\prime}} \log \left(\frac{2 R * H}{T_{d} * \Delta P_{L}^{\prime} * D^{\prime}+2 R * H}\right) .
$$

The value of frequency deviation at nadir can be obtained by substituting (7) into (6), and the maximum frequency deviation $\left|\Delta f_{\text {nadir }}\right|$ should not exceed the predefined threshold $\Delta f_{\max }$ :

$$
\begin{aligned}
\left|\Delta f_{\text {nadir }}\right|= & \Delta f_{\mathrm{DB}}+\frac{\Delta P_{L}{ }^{\prime}}{D^{\prime}} \\
& +\frac{2 R * H}{T_{d} * D^{\prime 2}} \log \left(\frac{2 R * H}{T_{d} * D^{\prime} * \Delta P_{L}{ }^{\prime}+2 R * H}\right) \\
\leq & \Delta f_{\max } .
\end{aligned}
$$

Rearranging (8) gives

$$
\begin{aligned}
\frac{2 R * H}{T_{d}} \cdot \log ( & \left.\frac{2 R * H}{T_{d} * D^{\prime} * \Delta P_{L}{ }^{\prime}+2 R * H}\right) \\
& \leq D^{\prime 2}\left(\Delta f_{\max }-\Delta f_{\mathrm{DB}}\right)-D^{\prime} * \Delta P_{L}^{\prime}
\end{aligned}
$$

Proposition: $\left|\Delta f_{\text {nadir }}\right| \leq \Delta f_{\max }$ if the following mixed integer linear constraints are satisfied (sufficient conditions):

$$
\left\{\begin{array}{l}
\frac{\sum_{g \in G_{\mathrm{CONV}}} H_{g} * P_{g}^{\max } * y_{g}-\Delta P_{L}^{\max } * H_{L}^{\max } * R}{f_{0}} \geq k^{*} \\
-M\left(1-N_{g}^{\mathrm{up}}\right) \leq y_{g}-R \leq M\left(1-N_{g}^{\mathrm{up}}\right) \\
-M * N_{g}^{\mathrm{up}} \leq y_{g} \leq M * N_{g}^{\mathrm{up}}
\end{array}\right.
$$

where $M$ is a large number and $k^{*}$ is the unique solution from

$$
\begin{aligned}
\frac{2 k^{*}}{T_{d}} \cdot \log \left(\frac{2 k^{*}}{T_{d} * D^{\prime} * \Delta P_{L}{ }^{\prime}+2 k^{*}}\right) \\
=D^{\prime 2}\left(\Delta f_{\max }-\Delta f_{\mathrm{DB}}\right)-D^{\prime} * \Delta P_{L}{ }^{\prime} .
\end{aligned}
$$

Proof: The left-hand side of inequality (9) is a monotonically decreasing function of $R * H(>0)$. Therefore, for any given value of $D^{\prime}, \Delta P_{L}$ and $\Delta f_{\mathrm{DB}}$, there exists a unique value of $R * H$, denoted by $k^{*}$, such that

$$
\begin{aligned}
\frac{2 k^{*}}{T_{d}} \cdot \log \left(\frac{2 k^{*}}{T_{d} * D^{\prime} * \Delta P_{L}{ }^{\prime}+2 k^{*}}\right) \\
=D^{\prime 2}\left(\Delta f_{\max }-\Delta f_{\mathrm{DB}}\right)-D^{\prime} * \Delta P_{L}{ }^{\prime} .
\end{aligned}
$$

Then condition $\left|\Delta f_{\text {nadir }}\right| \leq \Delta f_{\max }$ is satisfied if

$$
H * R \geq \mathrm{k}^{*} .
$$

The system inertia can be calculated by using $H=$ $\left(\sum_{g \in \mathcal{G}} H_{g} * P_{g}^{\max } * N_{g}^{\mathrm{up}}-\Delta P_{L}^{\max } * H_{L}^{\max }\right) /\left(f_{0}\right)$. Therefore, the requirement on frequency nadir can be formulated as bilinear constraint

$$
\frac{\left(\sum_{g \in \mathcal{G}} H_{g} * P_{g}^{\max } * N_{g}^{\mathrm{up}}-\Delta P_{L}^{\max } * H_{L}^{\max }\right) * R}{f_{0}} \geq k^{*} .
$$

By defining an additional variable $y_{g}$ and applying standard reformulation method as in [22], condition (14) can be transformed to MILP constraints as shown in (10).

\section{Frequency Level at Quasi-Steady-State}

The intermediate quasi-steady-state condition depends essentially on the total amount of PFR delivered by generators at the delivery time. We denote $\Delta f_{\max }^{\text {ss }}$ as the maximum allowed quasi-steady-state frequency deviation; hence, considering $\Delta P_{L}^{\max }$, this frequency deviation can be found, by assuming in (3), that RoCoF is effectively zero, i.e., that the frequency has reached a constant level:

$$
\left|\Delta f^{\mathrm{ss}}\right|=\frac{\Delta P_{L}^{\max }-R}{D * P^{D}} \leq \Delta f_{\max }^{\mathrm{ss}}
$$

This allows quantifying the required PFR to satisfy the quasisteady-state frequency criterion as

$$
R \geq \Delta P_{L}^{\max }-D * P^{D} * \Delta f_{\max }^{\mathrm{ss}}
$$

Unlike the other two constraints, the quasi-steady-state constraint does not depend on the level of system inertia.

\section{E. Fast Frequency Response Requirements in GB Future Low Carbon Scenario}

In the present GB system, the amount of PFR requirements is based on the demand level, which is primarily driven by the quasi-steady state frequency threshold. However, the increased rating of the largest plant and the growing penetration of wind generation will make constraints associated with transient frequency evolution significantly more relevant. In this subsection we demonstrate the change in PFR requirements from being determined by quasi-steady-state frequency limit, to being driven by nadir frequency limit. Assuming the inertia constant of $H_{g}=5 \mathrm{~s}$ and an average generators' loading level $(80 \%$ of the units' capacity), the present quasi-steady-state frequency driven PFR requirement (red in Fig. 4) is compared with the nadir frequency driven FPR requirement (black). At present, given the largest plant rating of $1.32 \mathrm{GW}$, PFR requirement driven by the quasi-steady-state frequency (red solid) is always binding, i.e., being above the frequency nadir driven requirement (black solid). This justifies the present requirements of

$$
|\Delta f(t)|= \begin{cases}\left(\frac{\Delta P_{L}^{\max }}{D^{\prime}}\right) \cdot\left(1-e^{-\frac{D^{\prime}}{2 H} t}\right) & \text { if } t<t_{\mathrm{DB}} \\ \Delta f_{\mathrm{DB}}+\left(\frac{\Delta P_{L}{ }^{\prime}}{D^{\prime}}+\frac{2 R * H}{T_{d} * D^{\prime 2}}\right) \cdot\left(1-e^{-\frac{D^{\prime}}{2 H} t^{\prime}}\right)-\frac{R * t^{\prime}}{T_{d} * D^{\prime}} & \text { if } t \geq t_{\mathrm{DB}}\end{cases}
$$




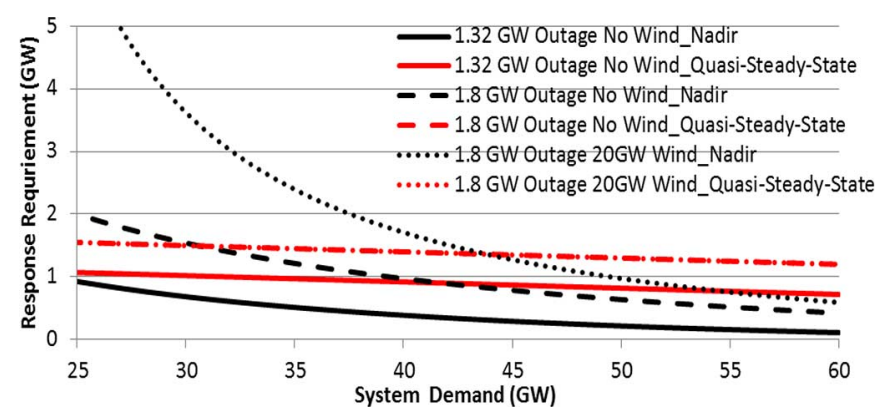

Fig. 4. Nadir (black) versus quasi-steady-state (red) response requirement.

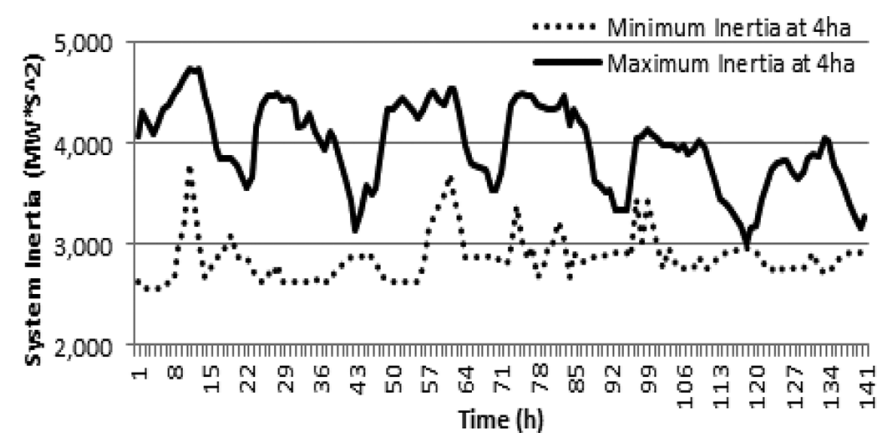

Fig. 5. Example of maximum (solid) and minimum (dotted) system inertia in 4-h ahead.

PFR described above. On the other hand, after the $1.8 \mathrm{GW}$ nuclear plant is commissioned, frequency nadir driven PFR requirement would dominate the overall requirements when demand is lower than $30 \mathrm{GW}$ in the system without wind (dashed) or when demand is lower than $45 \mathrm{GW}$ in the system with 20 GW wind output (dotted). Under those conditions, the present requirements may compromise the system security.

Another key concern is the impact of wind uncertainty on the scheduling of fast frequency response. The requirements of PFR depend on the system inertia, which will in turn be driven by the amount of synchronized conventional plant and the system demand. Different realizations of wind production could significantly change the schedule of conventional plants, resulting in different levels of system inertia.

Stochastic scheduling explicitly models the uncertainty in wind production by using the scenario tree. As shown in Fig. 1, commitment decisions are made in each node of the tree, based on the realization of wind production, which also provides the level of system inertia. In each time step, the system inertia could vary significantly depending on wind realization in each node of the scenario tree. Fig. 5 shows maximum (solid) and minimum (dotted) levels of system inertia in 4-h ahead. Although it is possible to select a conservative estimation of system inertia at each time step (always the minimum level); this would over-schedule fast frequency response, potentially degrading efficiency of system operation.

\section{CAse Study}

In this section, the proposed scheduling method is firstly compared against conventional methods. Then we demonstrate the impact of delivery time of PFR, the maximum RoCoF and the
TABLE I

Characteristics of Thermal Plants

\begin{tabular}{l|llll}
\hline & Nuclear & Coal & CCGT & OCGT \\
\hline Number of units & 6 & 40 & 70 & 30 \\
Rated Power $(\boldsymbol{M W})$ & 1800 & 500 & 500 & 200 \\
Min Stable Gen $(\boldsymbol{M W})$ & 1800 & 250 & 250 & 50 \\
No-load cost $(\mathbf{f} / \boldsymbol{h})$ & 0 & 3364 & 7809 & 8000 \\
Marginal cost $(\boldsymbol{f} / \mathbf{M W h})$ & 10 & 72 & 51 & 110 \\
Startup cost $(\boldsymbol{f})$ & $\mathrm{n} / \mathrm{a}$ & 32000 & 9000 & 0 \\
Startup time $(\boldsymbol{h})$ & $\mathrm{n} / \mathrm{a}$ & 6 & 4 & 0 \\
Min down time $(\boldsymbol{h})$ & $\mathrm{n} / \mathrm{a}$ & 4 & 4 & 0 \\
Inertia Constant $(\mathbf{s})$ & 5 & 5 & 5 & 5 \\
Max Response $(\boldsymbol{M W})$ & 0 & 75 & 75 & 40 \\
Response Slope & 0 & 0.3 & 0.4 & 0.6 \\
Emission(kgCO2/MWh) & 0 & 925 & 394 & 557 \\
Outage rate (occ/year) & 1.55 & 18 & 18 & $\mathrm{n} / \mathrm{a}$ \\
Mean time to repair(days) & 30 & 3 & 3 & $\mathrm{n} / \mathrm{a}$ \\
\hline
\end{tabular}

load damping rate on the operation cost and the ability of the system to absorb wind; the importance of recognizing different inertia capabilities of generators in UC is also discussed.

Simulations of annual system operation are performed using the GB 2030 scenario [23]. The maximum demand is 60 $\mathrm{GW}$, total conventional generation capacity is $70 \mathrm{GW}$ and the installed wind capacity is assumed to be $35 \mathrm{GW}(30 \%$ wind penetration). The annualized failure rate $\left(=8760 * \lambda_{g}\right)$ and mean time to repair $\left(=1 /\left(24 * \mu_{g}\right)\right)$ for each generation type are shown in Table I. Existing $2.6 \mathrm{GW}$ pump-hydro storage plant with $10 \mathrm{GWh}$ energy storage capacity and $75 \%$ round efficiency is also included in the generation mix. This storage plant can provide up to $500 \mathrm{MW}$ of PFR. Table I summarizes the characteristics of conventional plants [24]. The value of lost load (VOLL) is set at $30000 \mathcal{L}$ /MWh. The reference settings for delivery time $\left(T_{d}=10 \mathrm{~s}\right)$, frequency dead-band $\left(\Delta f_{\mathrm{DB}}=15 \mathrm{mHz}\right)$ and load-damping rate $(D=1 \% / \mathrm{Hz})$ are chosen according to the GB practice [13]. The proposed requirement on $\operatorname{RoCoF}(0.5 \mathrm{~Hz} / \mathrm{s})$ for the future $\mathrm{GB}$ system [18] is adopted. The optimization was solved by using FICO Xpress 7.1, which was linked to a $\mathrm{C}++$ simulation application via the BCL interface [25].

\section{A. Impact of Wind Uncertainty on System Inertia}

As shown in Fig. 5, the different realizations of wind power could lead to significant different levels of system inertia. Scenario-tree based stochastic scheduling model provides a platform to recognize the effect of unknown inertia caused by the wind uncertainty. The benefit of stochastic scheduling of reserve has been wildly recognized. This section explores the importance of scheduling fast frequency response by taking into account the impact of wind uncertainty on system inertia. Similar to the approach presented in [5] and [14], simulation of the system operation over one year time horizon is carried out involving 17520 time-steps. Analysis of the performance of different scheduling methods is based on the annual studies of the generation system operation.

1) Deterministic scheduling (DS): the scheduling is performed in the rolling basis, but only based on single scenario [5] with a quantile of 0.96 and the inertia-dependent fast frequency response requirement is calculated based on this single scenario. 
2) Stochastic scheduling with deterministic inertia $\left(S S \_1\right)$ : the traditional stochastic approach with 9 scenarios is applied, which is the same as the base case defined in [14]. The fast frequency response requirement is deterministic and conservative; it is calculated using the worst-case scenario, thus covering the minimum available system inertia in each time step.

3) Stochastic scheduling with explicitly considering the impact of wind uncertainty on system inertia $\left(S S_{\_} 2\right)$ : this scheduling method differs from SS_1 as, at each time step, the fast frequency response requirement is calculated for each scenario based on the associated system inertia.

The system performance with different methods is shown in Fig. 6. As expected, both stochastic methods reduce the operation costs and $\mathrm{CO}_{2}$ emission compared to the DS case. In particular, the $\mathrm{SS}_{-} 1$ provides approximately $1.2 \%$ operation cost reduction and $1 \%$ emission reduction, while the proposed method (SS_2) can further reduce the operation cost by more than $0.8 \%$ and the emission by more than $2 \%$.

Table II provides the details of system operation based on different scheduling methods. Compared with conservative approach (SS_1), directly considering the impact of wind uncertainty on system inertia allows to optimally scheduling high-cost but flexible plants (OCGT) to provide frequency response for the scenarios with low probability but very high response requirement. Therefore, the total spinning headroom is reduced, more wind generation is integrated and lower operation cost is achieved. It is also worth mentioning that the proposed method significantly reduces the energy supplied by highly-emitting coal plants; therefore $\mathrm{CO}_{2}$ emission of the system is dramatically reduced.

The computation time for each case is also presented in Table II. The half-hourly rolling planning simulation is carried out over the time horizon of one year, with a duality gap of $0.1 \%$. There are hence 17520 MILP optimizations in total. The case studies were carried out on a six-core Intel $3.46 \mathrm{GHz}$ Xeon processor with $12 \mathrm{~GB}$ RAM. It took about $1.6 \mathrm{~h}$ to solve the deterministic scheduling; while the computation times are much longer for both stochastic scheduling methods: SS_1 takes $23 \mathrm{~h}$ and SS_2 takes $27 \mathrm{~h}$.

The impacts of wind penetration level are also assessed (Fig. 7). When the wind penetration level is moderate, there is no significant economic benefit (difference between dotted and solid) from explicitly considering the impact of wind uncertainty on the system inertia. However, when the wind penetration level reaches $20 \%$ or above, significant operation cost saving can be obtained by using the proposed method.

\section{B. Delivery Time}

With a larger maximum plant rating and reduced inertia, frequency will achieve the nadir much faster; therefore, in order to contain this drop, governor response would need to be delivered faster. This sub-section illustrates the impact of PFR delivery time $T_{d}$ on the system performance. The maximum response capability and the slope (see Fig. 2) for each generator are assumed to remain the same as in Table I, but the delivery time is varied from $10 \mathrm{~s}$ to $3 \mathrm{~s}$. The results in Fig. 8 show an operation cost reduction (solid) by up to $3 \%$ from decreasing the

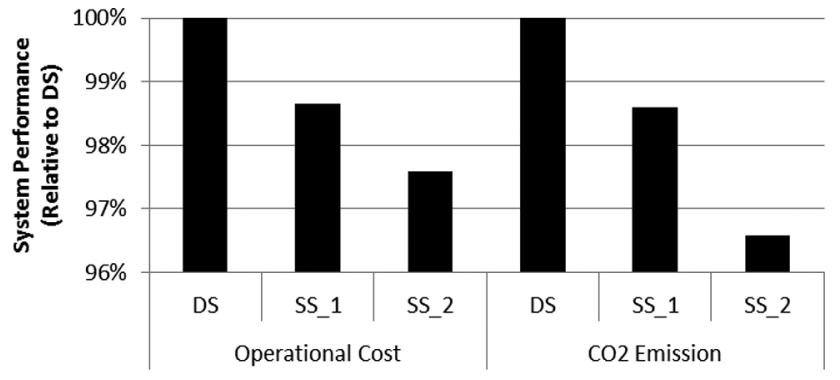

Fig. 6. System performance comparison under different scheduling methods.

TABLE II

DetAILED Results OF SySTEM OPERATION

\begin{tabular}{l|ccc}
\hline & DS & SS_1 & SS_2 \\
\hline Operation Cost (f/MWh) & 39.14 & 38.61 & 38.19 \\
Curtailed Wind (\% available wind) & 11.51 & 10.15 & 9.87 \\
Average spinning headroom (MW) & 10148 & 9583 & 8911 \\
COAL: Production (TWh) & 13.62 & 11.57 & 8.81 \\
COAL: Frequency Response (MW) & 387 & 297 & 241 \\
CCGT: Production (TWh) & 149.87 & 150.85 & 153.09 \\
CCGT: Frequency Response (MW) & 1602 & 1701 & 1781 \\
OCGT: Production (TWh) & 0.08 & 0.40 & 0.70 \\
OCGT: Frequency Response (MW) & 3 & 14 & 25 \\
Storage: Average state of charge (\%) & 92 & 71 & 65 \\
Storage: Frequency Response (MW) & 490 & 467 & 473 \\
Computation Time (hours) & 1.6 & 23 & 27 \\
\hline
\end{tabular}

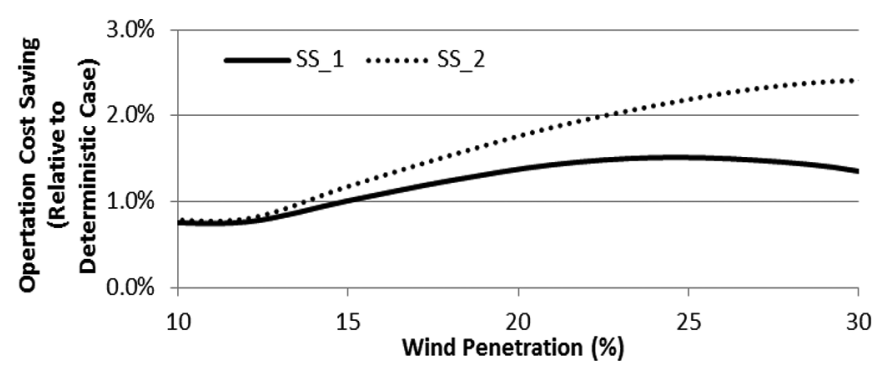

Fig. 7. Annual operation cost saving from different operation methods: SS_1 (solid) versus SS_2 (dotted).

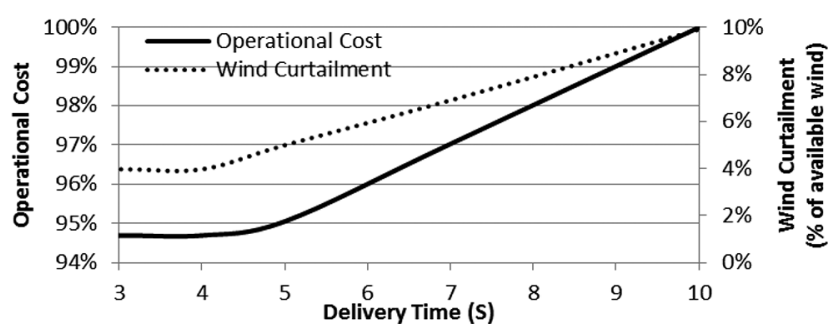

Fig. 8. Impact of reducing the delivery time on system operation cost (solid) and wind curtailment (dotted). The reference case is SS_2 case in Table II.

delivery time; in parallel, the amount of wind curtailed (dotted) reduces by up to $50 \%$. The benefits due to the reduction in delivery time show a clear saturation effect after $5 \mathrm{~s}$. This is because when the frequency delivery is fast enough to secure the nadir, the required additional power injection starts to be bounded by quasi-steady-state frequency requirement. These results are consistent with the discussion presented by National Grid [26]. 
This article has been accepted for inclusion in a future issue of this journal. Content is final as presented, with the exception of pagination.

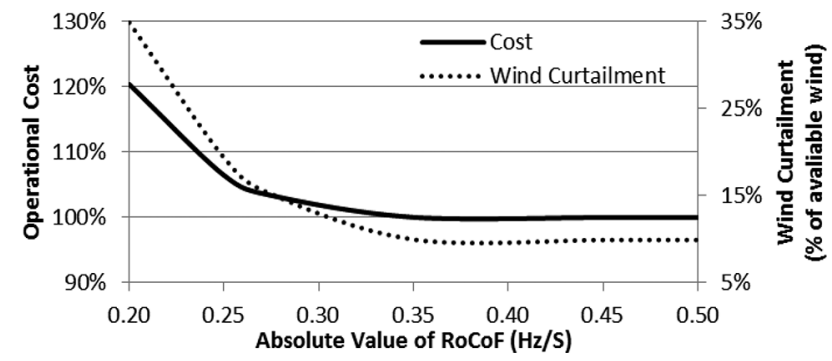

Fig. 9. Impact of maximum RoCoF setting on the operation cost (solid) and the wind curtailment (dotted). The reference case is SS_2 case in Table II.

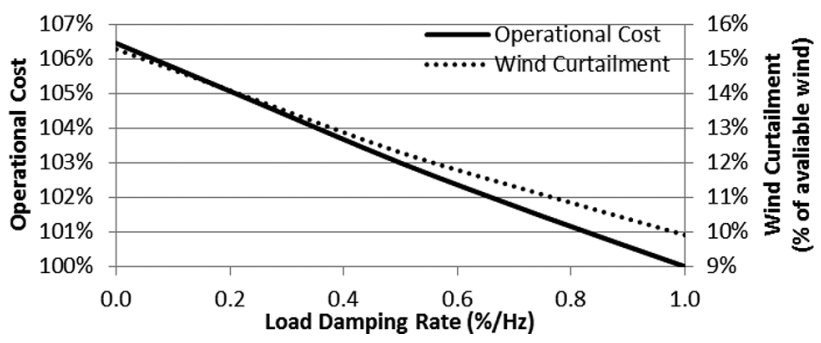

Fig. 10. Impact of load damping rate on the system operation cost (solid) and wind curtailment (dotted). The reference case is SS_2 case in Table II.

\section{RoCoF Setting}

Increase in RoCoF driven by reduced system inertia would force disconnection of distributed generation, increasing the system stress further. It is not clear yet how to choose appropriate RoCoF protection settings for the future GB system with high penetration of RES. Therefore, this section investigates the impacts of varying the maximum RoCoF from $0.5 \mathrm{~Hz} / \mathrm{s}$ to $0.2 \mathrm{~Hz} / \mathrm{s}$. As shown in Fig. 9, the $0.2 \mathrm{~Hz} / \mathrm{s}$ setting would lead to extremely high operation cost (solid) and wind curtailment (dotted). It is also worth noting that the benefits of relaxing maximum RoCoF beyond $0.4 \mathrm{~Hz} / \mathrm{s}$ will be limited. This conclusion supports the development of new recommendation to change the RoCoF protection settings for new and existing DG [18].

\section{Load Damping Rate}

This sub-section aims at investigating the impact of load damping rate on system operating costs and wind curtailment. As shown in Fig. 10, reducing load damping rate from $1 \% / \mathrm{Hz}$ to $0 \% / \mathrm{Hz}$ leads to an increase in operation cost (solid) up to $6.2 \%$ and an increase in wind curtailment from $7.5 \%$ to $10.7 \%$. Although the overall damping effect may decline in the future due to the increased use of power electronics interfaces, neglecting it in the scheduling process could increase the generation cost and limit the ability of the system to accommodate wind generation. In fact, it would be beneficial to stimulate alternative provision of damping effect.

\section{E. Recognition of Different Inertia Capability of Generators}

This sub-section demonstrates the benefits of recognizing the inertia capability of each generator in the scheduling process, which may inform the development of inertia-related markets, as proposed in [27]. For this purpose, $5 \mathrm{GW}$ of CCGT plant is assumed to be characterized by higher inertia constant $(8 \mathrm{~s})$ and
TABLE III

IMPACT OF INERTIA RECOGNITION ON THE ENERGy PRODUCTION

\begin{tabular}{l|cc}
\hline & $\begin{array}{c}\text { With Recognition of } \\
\text { Inertia (TWh) }\end{array}$ & $\begin{array}{c}\text { Without Recognition of } \\
\text { Inertia (TWh) }\end{array}$ \\
\hline Nuclear & 88 & 88 \\
CCGT & 135 & 156 \\
CCGT_High Inertia & 24.0 & 4.1 \\
COAL & 2.1 & 2.1 \\
CCGT & 0.5 & 0.5 \\
Absorbed Wind & 96.9 & 95.6 \\
\hline
\end{tabular}

also slightly higher marginal cost. Two simulations are carried out, one with and another without recognizing the inertia capability of the plants. Results in Table III suggest that if the inertia capability is explicitly considered, the scheduling process will commit more plants with higher inertia constant and their energy production will significantly increase, from around $4 \mathrm{TWh}$ to $24 \mathrm{TWh}$ in the study analyzed. We also observe an increase in the total operation cost when the inertia capability is not fully recognized in the scheduling process as this will lead to increase in the amount of part-loaded plant to respect the RoCoF and nadir constraints. By being able to exploit the high inertia capability of plant, a further $1.3 \mathrm{TWh}$ of wind can be integrated.

\section{SUMMARY AND DisCUSSION}

In this paper we propose a simplified system frequency evolution model and derive a set of MILP constraints in order to ensure that the system dynamic performance meets the security requirements. These constraints are then incorporated into a SUC model. This novel framework allows the impact of wind uncertainty on system inertia to be directly addressed, which avoids over-scheduling the frequency response. Numerical results show the benefits of the proposed method compared with traditional methods in terms of operation cost saving and wind curtailment reduction.

Moreover, the model proposed enables the impact that different settings of frequency response delivery time, RoCoF limit and load damping rate would have on the system operation cost and on the wind curtailment to be assessed. The results obtained regarding the RoCoF and delivery time can provide economic evidence to support appropriate reforms of the grid code. Furthermore, we demonstrate the value of recognizing different inertia capabilities of generators in the scheduling process.

There are several areas of enhancing the proposed framework. The developed model assumes a fixed delivery time for all the generators while considering different speeds of individual generators in delivering scheduled PFR will be important to provide appropriate incentives for speedy delivery of PFR. This becomes very relevant when fast frequency response resources, e.g., loads with under frequency relays or battery storage are integrated into the system since these could provide much faster frequency response when compared with conventional generation.

Furthermore, synthetic inertia from wind turbines is believed to play an important role in supporting the frequency performance in the future low carbon power system [28]. However, it is very challenging to incorporate synthetic inertia into a UC model, since there is uncertainty associated with aggregated 
synthetic inertia capability from wind turbines even for a given level of wind power production [29]. The proposed model could be potentially extended to incorporate these multiple uncertainties in the future.

\section{APPENDIX}

The equations given below summarize the proposed model for SUC with inertia-dependent frequency response requirement, which is based on the model in [14].

1. Objective function and system-level constraint The objective is to minimize the expected operation cost:

$$
\sum_{n \in N} \pi(n)\left(\sum_{g \in G} C_{g}(n)+\Delta \tau(n)\left(c^{\mathrm{LS}} P^{\mathrm{LS}}(n)\right)\right) .
$$

The load balance constraint can be written

$$
\begin{aligned}
\sum_{g \in G} P_{g}(n)+\sum_{s \in S}\left(P_{s}^{d}(n)-P_{s}^{c}(n)\right) & +P^{\mathrm{WN}}(n)-P^{\mathrm{WC}}(n) \\
= & P^{D}(n)-P^{\mathrm{LS}}(n) .
\end{aligned}
$$

The fast frequency response related constraints are

$$
\begin{aligned}
& H(n)=\frac{\sum_{g \in G} H_{g} * P_{g}^{\max } * N_{g}^{\mathrm{up}}(n)-\Delta P_{L}^{\mathrm{max}} * H_{L}^{\max }}{f_{0}} \\
& R(n)=\sum_{g \in G} R_{g}(n)+\sum_{s \in S} R_{s}(n) .
\end{aligned}
$$

RoCoF limit (A5), frequency nadir limit (A6), and quasi-steadystate frequency limit (A7) - see (A5) at the bottom of the page, where $M$ is a large number and $k^{*}(n)$ is the unique solution from

$$
\begin{aligned}
& \frac{2 k^{*}(n)}{T_{d}} \cdot \log \left(\frac{2 k^{*}(n)}{T_{d} * D^{\prime}(n) * \Delta P_{L}{ }^{\prime}+2 k^{*}(n)}\right) \\
& \quad=D^{\prime}(n)^{2}\left(\Delta f_{\max }-\Delta f_{\mathrm{DB}}\right)-D(n)^{\prime} * \Delta P_{L}{ }^{\prime} \\
& R(n) \geq \Delta P_{L}-D(n) * P^{D} * \Delta f_{\max }^{\mathrm{ss}} .
\end{aligned}
$$

2. Local constraints and cost functions for thermal plants Total power output and operating costs of generator $g$ is

$$
C_{g}(n)=c_{g}^{\mathrm{st}} N_{g}^{\mathrm{sg}}(n)+\Delta \tau(n)\left(c_{g}^{\mathrm{nl}} N_{g}^{\mathrm{up}}(n)+c_{g}^{m} P_{g}(n)\right) .
$$

Some of the constraints at node $n$ refer to subsets of the ancestors of $n . A_{g}^{\mathrm{st}}(n), A_{g}^{\mathrm{mu}}(n)$, and $A_{g}^{\mathrm{mo}}(n)$ is defined by (3), (4), and (5) in [14]

The power generation is limited by

$$
N_{g}^{\mathrm{up}}(n) P_{g}^{\mathrm{msg}} \leq P_{g}(n) \leq N_{g}^{\mathrm{up}}(n) P_{g}^{\max } .
$$

Generator $g$ that starts generating at node $n$ must be started up $T_{g}^{\text {st }}$ previously:

$$
N_{g}^{\mathrm{sg}}(n)=\sum_{a \in A_{g}^{\mathrm{st}}(n)} N_{g}^{\mathrm{st}}(a) .
$$

Generator $g$ that is generating at node $n$ must be generating at node $n$ ' $s$ parent or be started generating at node $n$, and also not been shut down at node $n$ :

$$
N_{g}^{\mathrm{up}}(n)=N_{g}^{\mathrm{up}}(a(n))+N_{g}^{\mathrm{sg}}(n)-N_{g}^{\mathrm{sd}}(n) .
$$

Generator $g$ that is off at node $n$ must be off at node $n$ 's parent or be shut down at node $n$, but also not been started up at node $n$ :

$$
N_{g}^{\text {off }}(n)=N_{g}^{\text {off }}(a(n))+N_{g}^{\text {sd }}(n)-N_{g}^{\text {st }}(n) .
$$

Generator $g$ that is allowed to be shut down at node $n$ must been generating at node $n$ 's parent, but also not been generating for less than $T_{g}^{\mathrm{mu}}$ hours:

$$
N_{g}^{\mathrm{sd}}(n) \leq N_{g}^{\mathrm{up}}(a(n))-\sum_{a \in A_{g}^{\mathrm{mu}}(n)} N_{g}^{\mathrm{sg}}(a) .
$$

Generator $g$ that is allowed to be started up at node $n$ must be off at node $n$ 's parent, but also not been off for less than $T_{g}^{\text {mo }}$ hours:

$$
N_{g}^{\mathrm{st}}(n) \leq N_{g}^{\mathrm{off}}(a(n))-\sum_{a \in A_{g}^{\mathrm{moo}}(n)} N_{g}^{\mathrm{sd}}(a) .
$$

Ramp rate limits can be modelled as

$$
\begin{aligned}
& P_{g}(n)-P_{g}(a(n)) \leq \Delta \tau(a(n)) \Delta P_{g}^{r u} N_{g}^{\mathrm{up}}(n) \\
& P_{g}(n)-P_{g}(a(n)) \geq-\Delta \tau(a(n)) \Delta P_{g}^{r d} N_{g}^{\mathrm{up}}(a(n)) .
\end{aligned}
$$

The primary frequency response limits:

$$
\begin{aligned}
0 & \leq R_{g}(n) \leq R_{g}^{\max } \\
R_{g}(n) & \leq f_{g}^{F}\left(N_{g}^{\mathrm{up}}(n) P_{g}^{\max }-P_{g}(n)\right) .
\end{aligned}
$$

3. Local constraints for storage plants

The constraints for storage unit $s$ at node $n$ are modeled as

$$
\begin{aligned}
E_{s}^{\min } & \leq E_{s}(n) \leq E_{s}^{\max } \\
0 & \leq P_{s}^{c}(n) \leq\left(1-N_{s}^{\mathrm{Gen}}(n)\right) P_{s}^{c \max } \\
0 & \leq P_{s}^{d}(n) \leq N_{s}^{\mathrm{Gen}}(n) P_{s}^{d \max } \\
E_{s}(n) & =E_{s}(a(n))+\Delta \tau(n)\left(\eta_{s}^{c} P_{s}^{c}(n)-\frac{P_{s}^{d}(n)}{\eta_{s}^{d}}\right) .
\end{aligned}
$$

$$
H(n) \geq\left|\frac{\Delta P_{L}^{\max }}{2 R o C o F_{\max }}\right| \times\left\{\begin{array}{l}
\frac{\sum_{g \in G} H_{g} * P_{g}^{\max } * y_{g}(n)-\Delta P_{L}^{\max } * H_{L}^{\max } * R(n)}{f_{0}} \geq k^{*}(n) \\
-M\left(1-N_{g}^{\mathrm{up}}(n)\right) \leq y_{g}(n)-R(n) \leq M\left(1-N_{g}^{\mathrm{up}}(n)\right) \\
-M * N_{g}^{\mathrm{up}}(n) \leq y_{g}(n) \leq M * N_{g}^{\mathrm{up}}(n)
\end{array}\right.
$$


The primary frequency response characteristics of pumpstorage unit are modeled as

$$
\begin{aligned}
0 & \leq R_{s}(n) \leq R_{s}^{\max } \\
R_{s}(n) & \leq\left(N_{s}^{\mathrm{Gen}}(n) P_{s}^{d \max }-P_{s}^{d}(n)+P_{s}^{c}(n)\right) .
\end{aligned}
$$

\section{REFERENCES}

[1] EirGrid and SONI, Facilitation of Renewables, 2010 [Online]. Available: http://www.eirgrid.com/renewables/facilitationofrenewables/

[2] E. M. Garrigle, J. Deane, and P. Leahy, "How much wind energy will be curtailed on the 2020 Irish power system?," Renew. Energy, no. 55, pp. 544-553, 2013.

[3] A. J. Conejo, M. Carrion, and J. M. Morales, Decision Making Under Uncertainty in Electricity Markets. New York, NY, USA: Springer, 2010.

[4] P. Meibom, R. Barth, B. Hasche, H. Brand, C. Weber, and M. O'Malley, "Stochastic optimization model to study the operational impacts of high wind penetrations in Ireland," IEEE Trans. Power Syst., vol. 26, no. 3, pp. 1367-1379, Aug. 2011

[5] A. Tuohy, P. Meibom, E. Denny, and M. O'Malley, "Unit commitment for system with significant wind penetration," IEEE Trans. Power Syst., vol. 24, no. 2, pp. 592-601, May 2009.

[6] A. Papavasiliou, S. S. Oren, and R. P. O'Neill, "Reserve requirements for wind power integration: A scenario-based stochastic programming framework," IEEE Trans. Power Syst., vol. 26, no. 4, pp. 2197-2011, Nov. 2011.

[7] A. Sturt and G. Strbac, "Value of stochastic reserve policies in lowcarbon power systems," Inst. Mech. Eng., Part O: J. Risk Rel., pp. $51-64,2012$.

[8] J. F. Restrepo and F. Galiana, "Unit commitment with primary frequency regulation constraints," IEEE Trans. Power Syst., vol. 20, no. 4, pp. 1588-1596, Nov. 2005.

[9] R. Doherty, G. Lalor, and M. O'Malley, "Frequency control in competitive electricity market dispatch," IEEE Trans. Power Syst., vol. 20, no. 3, pp. 1588-1596, Aug. 2005.

[10] H. Chavez, R. Baldick, and S. Sharma, "Governor rate-constrained OPF for primary frequency control adequacy," IEEE Trans. Power Syst., vol. 29, no. 3, pp. 1473-1480, May 2014.

[11] H. Ahmadi and H. Ghasemi, "Security-constrained unit commitment with linearized system frequency limit constraints," IEEE Trans. Power Syst., vol. 29, no. 4, pp. 1536-1545, Jul. 2014.

[12] Y. Lee and R. Baldick, "A frequency-constrained stochastic economic dispatch model," IEEE Trans. Power Syst., vol. 28, no. 3, pp. 2301-2312, Aug. 2013.

[13] National Grid, Security and Quality of Supply Standards [Online]. Available: http://www2.nationalgrid.com/UK/Industry-information/ Electricity-codes/System-Security-and-Quality-of-Supply-Standards/

[14] A. Sturt and G. Strbac, "Efficient stochastic scheduling for simulation of wind-integrated power systems," IEEE Trans. Power Syst., vol. 27, no. 3, pp. 323-334, Aug. 2012.

[15] A. Sturt and G. Strbac, "Time-series modelling of power output for large-scale wind fleets," Wind Energy, vol. 14, no. 8, pp. 953-966, 2011.

[16] A. Sturt and G. Strbac, "A time series model for the aggregate Great Britain wind output circa 2030," IET Renew. Power Gener., 2013.

[17] R. Billiton and R. Allan, Reliability Evaluation of Power System. New York, NY, USA: Plenum, 1996.

[18] Energy Networks Association and National Grid, Frequency Changes During Large Disturbances and Their Impact on the Total System, 2013.
[19] J. O'Sullivan, A. Rogers, D. Flynn, P. Smith, and M. O'Malley, "Studying the maximum instantaneous non-synchronous generation in an Island system-Frequency stability challenges in Ireland," IEEE Trans. Power Syst., vol. 29, no. 6, pp. 2943-2951, Nov. 2014

[20] P. Kundur, Power System Stability and Control. London, U.K.: McGraw-Hill.

[21] H. Huang and F. Li, "Sensitivity analysis of load-damping characteristic in power system frequency regulation," IEEE Trans. Power Syst., vol. 28, no. 2, pp. 1324-1335, May 2013.

[22] W. Qianfan, J.-P. Watson, and Y. Guan, "Two-stage robust optimization for contingency constrained unit commitment," IEEE Trans. Power Syst., vol. 28, no. 3, pp. 2366-2375, Aug. 2013.

[23] G. Strbac, M. Aunedi, D. Pudjianto, P. Djapic, F. Teng, A. Sturt, D. Jackravut, R. Sansom, V. Yufit, and N. Brandon, Energy Futures Lab, Imperial College London, "Strategic assessment of the role and value of energy storage systems in the UK low carbon energy future," 2012.

[24] M. Aunedi, P.-A. Kountouriotis, J. E. O. Calderon, D. Angeli, and G. Strbac, "Economic and environmental benefits of dynamic demand in providing frequency regulation," IEEE Trans. Smart Grid, vol. 4, no. 4, pp. 2036-2048, Dec. 2013

[25] FICO Xpress optimization suite [Online]. Available: http://www.fico. $\mathrm{com} /$ en/products/fico-xpress-optimization-suite

[26] National Grid frequency response working group, Frequency Response Report, 2013.

[27] E. Ela, V. Gevorgian, A. Tuohy, B. Kirby, M. Milligan, and M. O'Malley, "Market designs for the primary frequency response ancillary service-Part I: Motivation and design part," IEEE Trans. Power Syst., vol. 29, no. 1, pp. 421-431, Jan. 2014.

[28] W. Lei and D. Infield, "Towards an assessment of power system frequency support from wind plant-Modeling aggregate inertial response," IEEE Trans. Power Syst., vol. 28, no. 3, pp. 2283-2291, Aug. 2013.

[29] R. Doherty, A. Mullane, G. Nolan, D. J. Burke, A. Bryson, and M. O'Malley, "An assessment of the impact of wind generation on system frequency control," IEEE Trans. Power Syst., vol. 25, no. 1, pp. 452-460, Feb. 2010.

Fei Teng (S'12) received the B.S. degree from Beihang University, China, in 2009.

He is a research assistant at Imperial College London, U.K. His research interests include power system operation and integration of renewable energy.

Vincenzo Trovato $\left(\mathrm{S}^{\prime} 12\right)$ received the B.S. degree in electrical engineering from Politecnico di Bari, Italy, in 2008 and the M.S. degree in electrical engineering from Politecnico di Torino, Italy, in 2010. He is pursuing the Ph.D. degree at Imperial College London, U.K.

His research interests include demand side response and its impact on the power system control and economics.

Goran Strbac (M'95) is a Professor of electrical energy systems with Imperial College London, U.K. His research interests are in modeling and optimization of electricity system operation and investment, economic and pricing, and integration of new forms of generation and demand technologies. 